\title{
„Haben Sie bedacht, Herr Minister, daß wir einen Menschen verloren haben?"
}

\author{
Kriegerwitwen in Westdeutschland nach 1945
}

\section{Kriegerwitwen, Öffentlichkeit und politische Partizipation}

Nach dem Zweiten Weltkrieg lebten in der Bundesrepublik Deutschland rund eine Million Kriegerwitwen. Deren Existenz war von sozialen Problemen geprãgt, außerdem mussten sie Kriegserlebnisse und -verluste psychisch verarbeiten. Ihre Versorgung durch den Staat und ihre Lebensweise wurden offentlich diskutiert, ihre individuellen Schicksale zu einer politischen Angelegenheit. Mit welchen Strategien Kriegerwitwen selbst ihre Situation meisterten, ob und wie sie für ihre Belange eintraten, ist bisher kaum erforscht, obwohl die genderorientierte Partizipationsforschung seit langem fordert, „den Fokus auf die Inklusion von bislang ausgeschlossenen Themen, Sphären, Formen und Akteurinnen " zu richten'.

Im Folgenden soll deshalb untersucht werden, ob das Verhältnis von Kriegerwitwen $z u$ und deren Engagement in bestimmten Teilöffentlichkeiten politische Partizipation bedeutete. Der Begriff „Kriegerwitwe“ wird bewusst verwendet, da er in der zeitgenōssischen Diskussion der 1950er Jahre präsenter ist als die Ausdrücke „Kriegswitwe" oder „Soldatenfrau“. Die Verbindung des archaischen Ausdrucks „Krieger“ mit dem Wort „Witwe“ („die ihres Manns beraubte“), mit dem Frauen öffentlich "markiert" wurden, weist auf bis heute wirksame Bedeutungen, die in den spāten 1940er und in den 1950er Jahren mit diesem Begriff assoziiert waren und deren Wurzeln wesentlich früher als 1945 liegen.

\footnotetext{
I Vgl. Barbara Holland-Cunz, Demokratiekritik. Zu Staatsbildern, Politikbegriffen und Demokratieformen, in: Ruth Becker/Beate Kortendiek (Hrsg.), Handbuch Frauen- und Geschlechterforschung. Theorie, Methoden, Empirie, Wiesbaden 2004, S.467-475, hier S.470. In meiner Dissertation „Aus dem Rahmen gefallen? Kriegerwitwen im westlichen Nachkriegsdeutschland 1945 bis $1960^{\circ}$, die in Kürze erscheint, habe ich Witwen erstmals als historische Akteurinnen und ihren Umgang mit öffentlichen Debatten untersucht.
} 
Der Beitrag geht davon aus, dass es eine Pluralitāt von Öffentlichkeiten gibt, die der Dichotomie Öffentlichkeit/Privatheit gegenüberzustellen ist. Dadurch werden die Grenzverschiebungen zwischen beiden Sphären und die Vielfalt öffentlicher Rāume, die sich Frauen und Mãnner trotz ihres Ausschlusses aus bestimmten Foren der bürgerlichen Gesellschaft schufen, fassbar ${ }^{2}$. Eine Untersuchung solcher Räume setzt voraus, Öffentlichkeit und Privatheit als Sphären mit fließenden Übergängen zu begreifen und die Entstehung von Öffentlichkeit sehr früh anzusetzen ${ }^{3}$. Damit eröffnen sich auch neue Perspektiven darauf, wie Frauen und Männer politisch aktiv wurden - und zwar sowohl jenseits institutionalisierter Partizipations- als auch konventioneller Beteiligungsformen. Politische Partizipation ist institutionalisiert, wenn sie rechtlich verankert und geregelt ist, zum Beispiel in der Verfassung oder einer Gemeindeordnung. Überdies meint institutionalisierte Partizipation Beteiligung in Organisationen wie Gewerkschaften, Parteien oder Verbänden. Konventionelle Beteiligungsformen zeichnen sich dadurch aus, dass sie etabliert, weit verbreitet und relativ üblich sind, wie etwa die Beteiligung an Wahlen oder die Mitarbeit in einer Partei. Demgegenüber stehen Formen nichtinstitutionalisierter und unkonventioneller Partizipation, die auch die Grenzen des Legalen überschreiten können ${ }^{4}$.

Zwei Aspekte sind bezüglich der Kriegerwitwen von Interesse. Erstens: Welche Formen von konventioneller Partizipation gab es für und von Kriegerwitwen in den 1950er Jahren? Zweitens: Welche Rolle spielte die einzelne Akteurin, die nicht kollektiv, sondern individuell handelte? Kriegerwitwen stellten Öffentlichkeit her, wenn auch selten in medialer Form wie etwa in der Presse. Sie wurden zudem meist als Einzelpersonen aktiv, ohne als Teil einer organisierten Gruppe mit einer Stimme zu sprechen. Es stellt sich hier also die Frage, ob Partizipation nur kollektiv erfolgen kann und welches Verstāndnis von Partizipation es ermöglicht, den Fokus auf die Einzelne und deren Engagement zu richten.

2 Vgl. Elisabeth Klaus, Das Ôffentliche im Privaten - Das Private im Öffentlichen. Ein kommunikationstheoretischer Ansatz, in: Friederike Herrmann/ Margret Lünenborg (Hrsg.), Tabubruch als Programm. Privates und Intimes in den Medien, Opladen 2001, S. 15-35, hier S. 18.

3 So betont Klaus (ebenda, S.20), dass bereits die „interpersonellen, mūndlichen Ausdrucksformen und alltãglichen, flūchtigen Kommunikationsformen" in eine Definition von Öffentlichkeiten einzubeziehen seien.

${ }^{4}$ Vgl. Brigitte Geißel/Virginia Penrose, Dynamiken der politischen Partizipation und Partizipationsforschung - politische Partizipation von Frauen und Männern, in: http://web.fu-berlin.de/gpo/geissel_penrose.htm, S. 4. 


\section{Umgang mit einem Massenschicksal: Kriegerwitwen und Kriegsopferverbände}

Im Gegensatz zu anderen alleinstehenden Frauen waren Kriegerwitwen unmittelbar von den Maßnahmen der Kriegsopferversorgung betroffen - einem für die junge Bundesrepublik kostspieligen Politikfeld, das in den 1950er Jahren höchst umkämpft war. Diese Form der Versorgung war zunächst nicht bundeseinheitlich geregelt und blieb nach Einführung des Bundesversorgungsgesetzes 1950 und seiner späteren Novellen bis in die 1960er Jahre hinein umstritten $^{5}$. Sie kostete zwischen 1950 und 1989 insgesamt 312 Milliarden DM, die Zahl der Versorgungsberechtigten erreichte 1952 mit 4,4 Millionen ihren Hōhepunkt ${ }^{6}$. Diese Maßnahmen beeinflussten die Lebensumstānde der Witwen unmittelbar ${ }^{7}$. Viele Parteipolitikerinnen, oft selbst Kriegsopfer, waren in den Ausschüssen für Kriegsopferfragen engagiert und publizierten zum Thema. Prominente politische Repräsentantinnen sprachen auf Tagungen der Kriegsopferverbānde oder auf von den Parteien veranstalteten Hinterbliebenentreffen, so die Bundestagsabgeordnete Elisabeth Schwarzhaupt (CDU) oder die Staatssekretärin im Bundesinnenministerium Dorothea Frandsen (CDU). Große Frauenverbānde wie der Deutsche Frauenrat traten hier weniger in Erscheinung. Für sie stand die Lage der weiblichen Hinterbliebenen nicht im Mittelpunkt ihres politischen Interesses, während sich die Kriegsopferausschüsse der großen Parteien vorrangig mit der Lage der vorwiegend männlichen Beschädigten beschäftigten.

Die wichtigste politische Interessenvertretung der Hinterbliebenen waren die Kriegsopferverbānde, die nach 1945 von den Alliierten verboten worden waren, sich aber in den $1950 \mathrm{er}$ Jahren wieder etablierten. Der Verband der Kriegsbeschādigten, Kriegshinterbliebenen und Sozialrentner Deutschlands (VdK) war mit 1,5 Millionen Mitgliedern Mitte der 1950er Jahre der größte unter ihnen. Die Verbãnde waren bereits institutionalisiert, da sie nach 1945 auf ihren Strukturen der Weimarer Republik aufbauen und auf

5 Vgl. Vera Neumann, Nicht der Rede wert. Die Privatisierung der Kriegsfolgen in der frūhen Bundesrepublik. Lebensgeschichtliche Erinnerungen, Münster 1999, S. 131.

${ }^{6}$ Vgl. Lutz Wiegand, Kriegsfolgengesetzgebung in der Bundesrepublik Deutschland, in: Archiv für Sozialgeschichte 35 (1995), S. 71-90, hier S.81.

${ }^{7}$ Vgl. Wolfgang Rüfner/Constantin Goschler, Ausgleich von Kriegs- und Diktaturfolgen, soziales Entschädigungsrecht, in: Günther Schulz (Hrsg.), Geschichte der Sozialpolitik in Deutschland seit 1945, Bd.3: 1949-1957. Bewältigung der Kriegsfolgen, Rūckkehr zur sozialpolitischen Normalitāt, Baden-Baden 2005, S.687-777. 
konventionelle Formen politischer Einflussnahme zurückgreifen konnten: Sie beteiligten sich aktiv an den Diskussionen über das Bundesversorgungsgesetz, begleiteten dessen Novellen, kritisierten öfentlich deren praktische Umsetzung und vertraten ihre Interessen in den Medien. Auf der lokalen Ebene berieten Hinterbliebenenbetreuerinnen, oft selbst Witwen und meist ehrenamtlich tātig, die Frauen, sprachen in ihrem Namen bei Ämtern vor und leisteten praktische Hilfe ${ }^{8}$.

Wenn sich Hinterbliebenenvertreterinnen explizit äußerten, dann meist im Rahmen sozial-moralischer Diskussionen: Witwen wurden hier als tapfere, geplagte Opfer und als Frauen dargestellt, die ihre Situation unter großem Verzicht meisterten. Demnach verdienten sie eine angemessene Versorgung, ohne dass diese ihren Verlust wirklich hătte wettmachen können. Verhielten sich Witwen jedoch nicht gemäß dieses diskursiven Programms, wurden sie mit Bezug auf traditionelle Vorstellungen von „sittlicher" Witwenschaft und „Normalfamilie“ als vermeintlicher Garantie der "natürlichen Ordnung der Gesellschaft" verurteilt.

Die Verbände machten sich zum Sprachrohr der Kriegerwitwen und legten dabei großes Engagement an den Tag. Trotz hitziger Debatten wurde in den Verbānden jedoch viel über, aber wenig mit Witwen gesprochen und für sie und weniger mit ihnen politisch interveniert. Diese große Gruppe Frauen stand wie keine andere für den verlorenen Krieg, zugleich befand sie sich in den 1950er Jahren aufgrund der nichtehelichen Lebensgemeinschaften vieler Witwen, der so genannten Onkelehen, im Mittelpunkt einer grundsätzlichen Debatte um die Rolle der Frau und der "Normalfamilie“ als Grundlage der bundesdeutschen Gesellschaft ${ }^{9}$. Dennoch blieb diese Gruppe meist stumm.

Hanna Gerig, Kölner CDU-Stadtverordnete und somit eine konventionell partizipierende Vertreterin dieser Frauen, fragte in einem Artikel von 1959 deswegen: „Sind Witwen schlechtere Staatsbürger?" In ihrer Veröffentlichung beklagte sie das mangelnde politische Engagement der Witwen, das sich in der geringen Wahlbeteiligung bei Bundes- und Landeswahlen ausdrücke, stellte aber

${ }^{8}$ Vgl. Verband der Kriegs- und Wehrdienstopfer, Behinderten und Sozialrentner Deutschlands, Landesverband Hessen (Hrsg.), Chronik VdK Hessen. Ein Stück Nachkriegsgeschichte, Frankfurt a.M. 1989.

${ }^{9}$ Viele Witwen lebten unverheiratet mit einem Mann zusammen, da sie in einer neuen Ehe den Anspruch auf Hinterbliebenenrente verloren hătten. Die Kinder der Witwen sollen den Partner der Mutter oft „Onkel“ genannt haben. Nach Schätzungen gab es Mitte der 1950er Jahre bis zu 300000 solcher Beziehungen. Vgl. Neumann, Privatisierung der Kriegsfolgen, S. 150. 
auch fest, sie seien aufgrund ihrer schwierigen finanziellen Lage, der Überforderung durch Erwerbsarbeit, Haushalt und Kindererziehung sowie der dürftigen Kriegsopferpolitik verbittert. Sie würden zudem durch die Kriegsopferverbände und die Opposition gegen die Politik der Union mobilisiert: „Witwen können sich nicht ausreichend informieren, sie fallen schneller auf leere Gerüchte und Behauptungen herein. Mindestens sind sie unsicher geworden und dann gehen sie - verärgert-deprimiert - gar nicht zur Wahl. ${ }^{110}$

Witwen als politisch aktive Vertreterinnen ihrer Interessen kommen in Gerigs Vorstellung nicht vor. Zudem fehlte es ihr wie auch anderen Entscheidungsträgern an konkreten Informationen über die tatsāchlichen Lebensumstānde und Einstellungen der Witwen, die sich nicht in der Versorgungsstatistik niederschlugen. So ãußerte die Bundestagsabgeordnete Marta Schanzenbach, die auch dem SPD-Vorstand angehörte, auf einem Kongress ihrer Partei für Kriegshinterbliebene 1964: „Es ist sehr zu bedauern, daß über die Fragen und Probleme der alleinstehenden Frau und damit auch der Kriegerwitwe keine umfassenden genauen Angaben gemacht werden können. ${ }^{11}$ Hier sprachen Akteurinnen für und über eine Gruppe Frauen, von der sie wenig wussten, aber viel zu wissen glaubten.

Ganz selten finden sich Zeugnisse, in denen sich die Witwen selbst oder ihre direkten Fürsprecherinnen aüßerten. So schrieben im Juni 1959 Reprāsentantinnen der rund 600000 im VdK organisierten Witwen nach Abschluss einer Bundeshinterbliebenenkonferenz einen offenen Brief an Bundesarbeitsminister Theodor Blank. Sie waren unter anderem darüber entrüstet, dass in der Novelle des Bundesversorgungsgesetzes von 1959 Nebeneinkommen, die Kriegerwitwen neben ihrer Grundrente in bestimmter Höhe dazu verdienen durften, zur Hälfte auf die Ausgleichsrenten angerechnet werden sollten. Dies bedeute, den Frauen die gleichzeitig beschlossene Erhöhung der Ausgleichsrente de facto wieder wegzunehmen. Zudem sei man nach wie vor mit der Hōhe der Grundrenten unzufrieden. Am Ende des Briefs heißt es:

${ }^{10}$ Hanna Gerig, Sind Witwen schlechtere Staatsbürger?, in: Frauen und Politik. Mitteilungen und Berichte der Christlich-Demokratischen Union 10 (1959), S.5-11, hier S.9.

11 Marta Schanzenbach, Die gesellschaftliche Stellung der Kriegerwitwe, in: Vorstand der SPD (Hrsg.), Gerechtigkeit für Hinterbliebene. Eine Dokumentation vom Kriegshinterbliebenenkongre $B$ der SPD am 28. und 29. Februar 1964 in der Festhalle Harmonie zu Heilbronn, Bonn 1964, S. 11-24, hier S.11. 
„Haben Sie bedacht, Herr Minister, daß wir einen Menschen verloren haben, einen Menschen, der nach christlicher Auffassung in der Waagschale irdischer und ewiger Bewertungen schwer wiegen sollte. Seit Jahren üben wir bitteren Verzicht auf Vieles, das anderen selbstverständlich ist und das keineswegs nur auf materiellem Gebiet. Wir finden es unwürdig, daß man mit uns, noch 14 Jahre nach Kriegsende, über die Gräber hinweg feilscht. Nicht nur einzelne Egoisten, auch der Staat nimmt es gedankenlos hin, daß wir unter großen Mühen unsere Kinder zu guten Staatsbürgern herangezogen haben. Schon sind es unsere Söhne, die zum Wehrdienst einberufen werden und wir als Mütter haben immer noch nicht die Anerkennung gefunden, die der Würde und Größe unseres Opfers entspricht.." 12

Die Argumentation der Schreibenden verdeutlicht, dass die Kriegsopferverbānde immer eine angemessene finanzielle Versorgung der Hinterbliebenen favorisierten. Kriegerwitwen durch Erwerbsarbeit sozial zu sichern, stand nie zur Debatte. Einerseits schloss man hier an die Tradition der Interessenpolitik der Weimarer Zeit an. Andererseits grenzte man sich gegenüber dem Osten Deutschlands und der sozialistischen Maxime ab, Frauen in die Erwerbsarbeit zu bringen. Die Diskussion über die Zuverdiengrenzen verweist jedoch darauf, dass die Grundrenten zu niedrig waren und die meisten Witwen arbeiten gehen mussten, um ihren Lebensunterhalt zu sichern. Das Problem der Vereinbarkeit von Erwerbsarbeit und Kinderbetreuung wird indirekt ebenfalls angesprochen. Die Argumentation greift jedoch auf das gängige Muster zurück: Opfer, Verlust, Verzicht, tapferes Meistern des Schicksals und Anspruch auf angemessene finanzielle Entschädigung.

\section{Kriegerwitwen als Akteurinnen: Eigensinn und konventionelle Partizipation}

Ohne das Engagement der Verbände und der dort organisierten Frauen zu schmälern, zeigen sich in der konkreten Situation einzelner Witwen große Kontraste zwischen dem, was die Verbānde forderten, und der Selbstwahrnehmung dieser Frauen. Neben den bereits genannten Problemen war folgender Konflikt für die Witwen zentral: Ihr Privatleben war wenigstens teilweise öffentlich, die Frauen standen aufgrund der finanziellen Abhängigkeit vom Staat

12 Zur Neuordnung des Bundesversorgungsgesetzes. Abdruck eines offenen Briefes der organisierten Kriegerwitwen im VdK an Bundesarbeitsminister Blank von 1959, in: Informationen für die Frau 6 (1959), S. 6. 
immer unter Beobachtung und waren - besonders wenn sie zusätzlich Hilfen der öffentlichen Fürsorge erhielten - immer wieder gezwungen, ihre Hilfsbedürftigkeit nachzuweisen. Obwohl die meisten Witwen als Haushaltsvorstannde fungierten und neben ihren eigenen Kindern oft Angehörige ihrer Herkunfts- und Schwiegerfamilie betreuten, also versorgende Frauen waren, wurden sie qua Gesetz als hinterbliebene Ehefrauen und damit als versorgte Frauen angesehen. Da Ehe und "Normalfamilie" wichtige Grundlagen des gesellschaftlichen Selbstverständnisses bildeten, waren Kriegerwitwen sowohl einer Familienpolitik mit inhärenter Geschlechterhierarchie als auch einem Versorgungsrecht unterworfen, das sie als hinterbliebene Ehefrauen behandelte. Ihr (sozialrechtlicher) Status wurde nur über den verstorbenen Ehemann definiert. Führten diese Frauen eine nichteheliche Beziehung, stand dies dem öffentlich geforderten Verhalten entgegen: Sie sollten entweder tapfere, verzichtende und versorgte Witwen sein oder wieder heiraten - nicht zuletzt, um die öffentlichen Kassen zu entlasten.

Die Witwen reagierten auf diesen sozialen und gesellschaftlichen Druck auf zweifache Weise: Erstens mit Eigensinn ${ }^{13}$ gegenüber den Behörden, indem sie bestimmte Fakten verschwiegen, sich bestimmten Maßnahmen entzogen oder ihnen mit offenem Widerstand begegneten. Zweitens: Mit einer bereits etablierten Form der Partizipation, also einer Eingabe beziehungsweise einem Bittgesuch bezüglich ihrer "Onkelehe" an den Bundesfamilienminister FranzJosef Wuermeling. In einer Sammlung von 50 Eingaben schrieben Kriegerwitwen über ihre persönliche Situation in einer nichtehelichen Lebensgemeinschaft, den finanziellen Zwang, aus dem heraus sie keine neue Ehe eingingen, über die Diffamierung durch Familie und Nachbarn sowie über die Probleme mit den eigenen Kindern. Wünsche nach konkreter Hilfe wurden geäußert und strategisch begründet, indem man eine neue Eheschließung als einzig anzustrebende Lösung des Problems darstellte, was jedoch an den Umständen scheitere. Die Witwen schrieben aber jede für sich und äußerten sich in diesem Rahmen genauso wenig kollektiv wie in den Verbānden. Das Leben in einer "Onkelehe“ war jedoch aufgrund der familienpolitischen Präferenzen der Regierung Adenauer und aufgrund der gesellschaftichen Wahrnehmung Mitte der 1950er Jahre ein Politikum. Sind also die Eingaben der Witwen schon Partizipation?

13 Zum Begriff vgl. Alf Lüdtke, Geschichte und Eigensinn, in: Berliner Geschichtswerkstatt (Hrsg.), Alltagskultur, Subjektivitåt und Geschichte. Zur Theorie und Praxis von Alltagsgeschichte, Münster 1994, S. 139-153. 
Eine nichteheliche Lebensgemeinschaft war für die Witwen, die an das Ministerium schrieben, keine selbstbewusst gewählte Lebensform, sondern die Folge der Lebensumstände. Außerdem zeigte sich, dass die „Onkelehe“ zwischen zwei Polen oszillierte: Anpassung einerseits und Eigensinn andererseits. Die Witwen distanzierten sich damit zwar von der Ehe als der von der Öffentlichkeit gewünschten Lebensform, verstanden dies jedoch nicht als einen bewussten Akt. Der Wunsch, mit einem Partner zu leben und dadurch Entlastung und emotionalen Rückhalt zu erfahren, war so stark, dass die Frauen soziale Sanktionen in Kauf nahmen, obwohl sie darunter litten.

Darüber hinaus reflektierten manche Witwen in den Briefen die offentliche Debatte um die "Onkelehe“ und äußerten ihr Unverstāndnis über die Beurteilung ihrer Situation, wobei ihr Blick über ihre eigene Befindlichkeit hinausging. So äußerte sich die Witwe F., Lehrerin und mehrfache Mutter, 1954 höchst kritisch zur Frage einer erneuten Ehe und zwar aufgrund der steuerlichen Bevorzugung von Ehepaaren, welche die geringfügige, aber durchaus bestehende wirtschaftliche Autonomie von Frauen aufhebe:

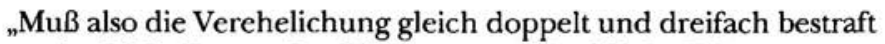
werden? Mit Entzug der Witwenrente, Wegfall der Witwensteuerermäßigung und nun auch noch durch eine gemeinsame Veranlagung die Arbeit der Verheirateten dermaßen entwertet werden? [...] Man kann nicht durch Gesetze, die Verhāltnisse ignorieren, die vom einschneidensten Krieg unserer Geschichte geschaffen wurden, diese bestehenden Verhāltnisse aus dem Weg schaffen." ${ }^{" 14}$

Die Witwe Hanna G., die als Mutter von drei Kindern mit einem Rentner zusammenlebte, verwies 1955 auf das Recht von Witwen, ihre Lebensform selbst zu bestimmen:

„Vieles wird uns, die wir eine so genannte ,Onkelehe‘ führen, verübelt. Aber können wir dafür, dass uns die Männer genommen wurden und dass die augenblicklichen Verhältnisse eine Wiederverheiratung erschweren? Sollen wir deshalb auf uns alleine gestellt bleiben und keinerlei Anspruch mehr an das Zusammensein und das Leben stellen dürfen?“

Frau G. machte die „Legalisierung“ ihrer nichtehelichen Lebensgemeinschaft offen davon abhängig, welchen Ausgleich sie und ihr Partner für ihre finanziellen Kriegsverluste erhielten und ob ihre

14 BA Koblenz, B 153/1113, Bundesministerium für Familienfragen, Briefe und Eingaben zur "Onkelehe“, Bl.23f. Die folgenden Zitate Bl.63 und Bl. 64 . 
finanzielle Situation im Fall einer erneuten Ehe gesichert sei: „Wenn dieses alles realisiert werden könnte, dann sind wir gerne bereit, das Zusammenleben zu legalisieren, zumal es sich hier um Werte handelt, die jedem einzelnen rechtmäßig gehört haben."

Ein weiteres Beispiel ist ein Brief der Witwe D. an die Frauenbeauftragte der Inneren Mission. Sie sei Kriegerwitwe, habe Kinder, lebe allerdings nicht in "wilder Ehe“, da sie die Ehe durchaus als eine "Lebensgemeinschaft bis zum Tod" ansehe. Sie könne, selbst wenn sie wolle, jedoch sowieso nicht wieder heiraten, da die finanziellen Belastungen für einen neuen Ehemann bei Wegfall der Witwenrente zu hoch wären. Sie argumentierte:

„Welchem Mann soll ich zumuten, meine Kinder zu ernãhren und zu kleiden? Das kann ich auch von einem Mann mit sehr gutem Einkommen nicht verlangen, denn die ehemalige Witwe gerăt dadurch in starke Abhängigkeit und ihr werden vielleicht bei der ersten Meinungsverschiedenheit die Kinder vorgeworfen. Auch wenn es ihr der Mann nicht direkt ins Gesicht sagt, weiß sie es trotzdem und wird nie ganz frei sein. So empfinde ich und mit mir andere Witwen auch."

Frau D. pochte andererseits darauf, Witwen das Recht auf eine "Onkelehe“ zuzugestehen: „Wenn sich also eine Witwe entschlieBt in wilder Ehe zu leben, hat niemand das Recht, mit Fingern auf sie zu zeigen, solange die ungerechte Berentung aufrechterhalten wird. ${ }^{\text {15 }}$

Witwen waren, das wird trotz dieser durchaus selbstbewussten Äußerungen deutlich, in ihrer Argumentation geprägt vom herrschenden Diskurs um Ehe und Normalfamilie. Ihre Schreiben entstanden aus dem Wunsch, die individuelle Lage zu verbessern. Jedoch machten sie damit ihre private Situation, die eine politische geworden war, durch eine Eingabe an eine Behörde oder ein Ministerium öffentlich und partizipierten auf diese Weise an einer Debatte über sich. Und ein weiterer Aspekt ist zu bedenken: Zwar schrieben die Witwen Mitte der 1950er Jahre einzeln und nicht kollektiv. Im Ministerium dürfte dies aber ganz anders wahrgenommen worden sein. Gerade die Vielzahl einzelner Briefe verlieh den Eingaben eine andere Qualität.

15 ADW, HGSt 794, Brief der Witwe D. an die Frauenbeauftragte der Inneren Mission Dr. H. Becker vom 1.7.1952. 


\section{Politische Partizipation oder individuelle Tellhabe?}

Es existierte außerhalb bereits etablierter Foren der Interessenvertretung für Kriegsopfer und Kriegshinterbliebene in den 1950er Jahren kein Raum, in dem Kriegerwitwen mit eigener Stimme sprachen und kollektiv eine „Gegenöffentlichkeit“ entwickelten ${ }^{16}$. Ihr Leben in nichtehelichen Lebensgemeinschaften hatte aber politische Folgen, da die Witwen damit die Ehe und die „Normalfamilie" als Grundlagen des bundesdeutschen Staats in Frage stellten. Die Rechtfertigung einer „Onkelehe“ bei gleichzeitiger „diskursiver" Partizipation ${ }^{17}$ war jedoch keine politische: Hier ging es um Handlungen, die nicht intentional, sondern in ihren Konsequenzen politisch waren ${ }^{18}$. Ungeachtet dessen zeigt sich im Fall der Kriegerwitwen, in welcher Weise Eigensinn diskursive und damit gesellschaftliche Teilhabe bedeuten konnte. Kriegerwitwen bewegten sich in ihrer Argumentation zwar nicht über den traditionellen Rahmen hinaus und sprachen nicht mit einer "gegenöffentlichen" Stimme. Aber ihre „eigene" Stimme war vorhanden, wenn auch nicht in Form kollektiver politischer Partizipation.

Erst in den 1960er Jahren profilierten sich die in den Verbānden organisierten Kriegerwitwen stärker in den institutionalisierten Partizipationsforen. Neben Versorgungsfragen spielten zunehmend Probleme des Alterns eine Rolle, die auch andere āltere Frauen und Rentnerinnen betrafen. Es wäre untersuchenswert, inwieweit das verānderte politische Klima der 1960er Jahre und die Kritik der Neuen Frauenbewegung an den bestehenden Geschlechterverhältnissen die Interessenvertretung der Kriegerwitwen in den Verbānden beeinflusste. Allerdings blieben auch die älter gewordenen, organisierten Kriegerwitwen, selbst wenn sie sich jetzt stärker öffentlich artikulierten, bei ihrer Partizipation im Rahmen des Institutionalisierten. Hier wurde mehr repräsentiert als von einer breiten Basis von Kriegerwitwen politisch partizipiert.

16 Vgl. Nancy Fraser, Öffentlichkeit neu denken. Ein Beitrag zur Kritik real existierender Demokratie, in: Elvira Scheich (Hrsg.), Vermittelte Weiblichkeit. Feministische Wissenschafts- und Gesellschaftstheorie, Hamburg 1996, S. 151-182, hier S. 166 und S. 172f.

17 Bettina Westle, Politische Partizipation und Geschlecht, in: Achim Koch/Martina Wasmer/Peter Schmidt (Hrsg.), Politische Partizipation in der Bundesrepublik Deutschland. Empirische Befunde und theoretische Erklārungen, Opladen 2001, S. 131-168, hier S. 137; „diskursive Partizipation“ meint Teilhabe an einer Debatte, die eine persönliche Lage betrifft.

18 Vgl. Max Kaase, Politische Beteiligung, in: Martin Greiffenhagen/Sylvia Greiffenhagen (Hrsg.), Handwörterbuch zur politischen Kultur der Bundesrepublik Deutschland, Wiesbaden 2002, S.349-355, hier S.350. 